\title{
Is Dipstick Urinalysis Screening Beneficial in Men with Lower Urinary Tract Symptoms?
}

Franklin C. Lowe - Martin C. Michel (D) · Jan M. Wruck •

Anna E. Verbeek

Received: June 11, 2019 / Published online: August 7, 2019

(C) The Author(s) 2019

\section{ABSTRACT}

Introduction: Dipstick urinalysis is a widely used screening tool in the evaluation of men with lower urinary tract symptoms (LUTS) suggestive of benign prostatic hyperplasia (BPH). As limited data support the use of dipstick urinalysis, we have used data from three recently published studies to assess clinical outcomes in those who had dipstick urinalysis findings for blood, glucose, and/or leukocytes.

Enhanced Digital Features To view enhanced digital features for this article go to https://doi.org/10.6084/ m9.figshare.8983973.

Electronic Supplementary Material The online version of this article (https://doi.org/10.1007/s12325019-01042-0) contains supplementary material, which is available to authorized users.

\section{F. C. Lowe}

Montefiori Medical Center, Albert Einstein College of Medicine, Bronx, NY, USA

M. C. Michel ( $\square)$

Department of Pharmacology, Johannes Gutenberg University, Obere Zahlbacher Str. 67, 55131 Mainz, Germany

e-mail: marmiche@uni-mainz.de

J. M. Wruck · A. E. Verbeek

Boehringer Ingelheim Pharmaceuticals, Inc., Ridgefield, CT, USA

A. E. Verbeek

Sanofi Consumer Health Care, Bridgewater, NJ, USA
Methods: We analyzed data from three observational studies involving men interested in using over-the-counter tamsulosin: a self-selection study (SSS) and two actual-use studies of 8-week (AUS8) and 24-week (AUS24) durations. Subgroup analyses focused on pooled data from participants not using $\alpha$-blockers or other prescription medication for LUTS suggestive of BPH (nonRx users) and who had urine dipstick findings. Data from participants using $\alpha$-blockers (AUS8) or any prescription BPH medications (SSS and AUS24) are presented as reference.

Results: Overall, 2488 nonRx users underwent dipstick urinalysis and 680 (27.3\%) had positive findings including traces of blood (332; 13.3\%), glucose $(259 ; 10.4 \%)$, and/or leukocytes (245; 9.8\%). Among users of prescription medicines, $21.6 \%(37 / 171)$ in SSS, $27.4 \%(23 / 84)$ in AUS8, and $31.1 \%(47 / 151)$ in AUS24 had urine dipstick findings. The 200 dipstick-positive nonRx users in SSS underwent per protocol urological assessment: $26(13.0 \%)$ had a newly diagnosed condition causing/contributing to urinary symptoms of which $2.9 \%$ were identified as medically important conditions. Among nonRx users with or without a dipstick finding, medically important conditions reported included prostate cancer $(1.0 \%$ vs. $1.0 \%$, respectively) and urolithiasis $(1.0 \%$ vs. $0.3 \%$, respectively). The proportion of men with dipstick urinalysis findings was similar between men who regularly visited their physician and those who did not. 
Conclusion: Dipstick urinalysis did not markedly increase the detection of undiagnosed medically important conditions that cause/contribute to urinary symptoms, suggesting that this test may not be a very effective screening tool for men with LUTS.

Funding: Boehringer Ingelheim Pharmaceuticals, Inc.

Keywords: Benign prostatic hyperplasia; Dipstick; Lower urinary tract symptoms; Urinalysis; Urology

\section{INTRODUCTION}

Male lower urinary tract symptoms (LUTS) are often attributed to benign prostatic hyperplasia/ enlargement (BPH/BPE) but can result from a broad range of other etiologies, including prostate and bladder cancer, urinary tract infections, urolithiasis, or systemic conditions such as diabetes mellitus $[1,2]$. While early intervention is recommended to improve patient outcomes and quality of life [3], causes of male LUTS other than BPH/BPE may need different types of treatment. Failure to recognize and treat alternative causes may have serious consequences because some of the alternative causes can lead to serious morbidity if left untreated. Therefore, guidelines for the management of male LUTS, such as those by the American Urological Association (AUA) [1] and the European Association of Urology [2], recommend that health care providers (HCPs) conduct a basic evaluation prior to initiating treatment. Such evaluation includes obtaining relevant patient history; assessing symptoms and bother using validated quantitative assessment tools; conducting physical examination, including a digital rectal exam; determining serum prostate-specific antigen levels; and performing dipstick urinalysis [1, 2]. Although guidelines recommend dipstick urinalysis in patients with LUTS to detect potential hematuria, including asymptomatic microscopic hematuria $(\mathrm{AMH})$, pyuria, urinary tract infection, and diabetes mellitus $[1,2]$, the value of this test as a screening tool is based on limited evidence and has been questioned [4].
False-positive results of dipstick urinalysis may occur for a variety of reasons, such as the presence of various substances in the urine [5], variability in collection technique (e.g., patients not retracting foreskin when providing sample) [6], and the methodology used to read the test strips (e.g., manual visual vs. automated machine reading). Moreover, similar to many screening tests, dipstick urinalysis has limited specificity. Based on a meta-analysis, the specificity of urine dipstick testing for leukocyte esterase was 17-93\% [7]. The detection of hematuria by dipstick testing is based on peroxidase activity catalyzed by erythrocytes, which may also be catalyzed by myoglobin and hemoglobin in the blood, leading to false-positive results [5]. Therefore, it is generally recommended that a dipstick finding of blood should be validated by microscopic analyses $[5,8]$. In the event of conflicting dipstick urinalysis (positive) and microscopic examination (negative) results, three additional repeat microscopic tests are required; at least one positive repeat test necessitates further workup [8]. False-positive and false-negative dipstick urinalysis results are common [5]. Accordingly, evaluation of $\mathrm{AMH}$ should be based solely on findings from microscopic urine examination and not on dipstick urinalysis results according to the applicable AUA guideline [8].

To obtain more evidence on the value of dipstick urinalysis, we analyzed three recently reported studies that were carried out to evaluate the feasibility of offering an over-the-counter (OTC) tamsulosin option to men interested in self-directed care for their bothersome urinary symptoms [9-11]. On the basis of advice by the Food and Drug Administration, dipstick urinalysis was conducted as a safety measure to screen for medically important conditions (MICs), in an attempt to limit the delay in diagnosis or treatment of LUTS causes other than BPH/BPE in OTC tamsulosin users. These studies included a self-selection study (SSS) and two actual-use studies. Dipstick urinalysis was performed in men who expressed interest in using OTC tamsulosin or who purchased and used the product in a self-directed manner. We aimed to assess and characterize potential clinical outcomes in participants who had a urine 
dipstick finding of a trace or more of blood, glucose, and/or leukocytes.

\section{METHODS}

\section{Study Design}

We analyzed data from three observational studies involving men with a urine dipstick finding at study baseline: an SSS (no medication being dispensed) with urological assessment of predefined subpopulations [10]; an exploratory, 8-week actual-use study with OTC tamsulosin (AUS8; NCT01726270) [9]; and a 24-week actual-use study with OTC tamsulosin (AUS24; NCT02573311) [11]. Detailed methodologies of these studies have been described previously; each of them had been approved by an independent ethical committee [9-11]. In brief, eligible men (age $\geq 18$ years) interested in selfmanaging their urinary symptoms reviewed an OTC tamsulosin product and determined suitability of its use. Men who believed the product was right for them to use or who were interested in purchasing it underwent dipstick urinalysis. In SSS, men who had a trace or more of blood, glucose, and/or leukocytes on dipstick urinalysis were referred to a board-certified urologist in their area to undergo full urological assessment, while in AUS8 and AUS24, men with these dipstick findings were informed of these findings and referred to their regular health care providers for follow-up and the outcomes were recorded. Men with dipstick findings in AUS8 and AUS24 were eligible to purchase and use OTC tamsulosin in a self-directed manner. In AUS8, only men who were not using an $\alpha$ blocker at study start (nonRx users) were eligible to purchase and use OTC tamsulosin, whereas in AUS24, two cohorts were enrolled: cohort 1 included interested men not currently using a prescription medication for BPH (nonRx users), and cohort 2 included interested men who were current users of such medications ( $\mathrm{Rx}$ users). Figure 1 shows a flowchart of the three studies. All men in these studies provided written informed consent for study participation. The studies were conducted in compliance with the protocol, the principles in the Declaration of
Helsinki, the International Conference on Harmonisation Harmonised Tripartite Guideline for Good Clinical Practice, and relevant Boehringer Ingelheim Standard Operating Procedures. Informed consent was obtained from all individual participants included in the studies. The studies were approved by independent ethics committees which are detailed in the previous publications.

\section{Baseline Assessments}

Participant age; duration and severity of urinary symptoms (defined by AUA-Symptom Index); occurrence of other noteworthy symptoms; frequency of, timing of, and discussions with HCPs; presence of targeted medical conditions; and dipstick urinalysis findings were collected at baseline for each study. Targeted medical conditions comprised urological conditions that might be observed with the presence of male LUTS and commonly reported nonurological conditions in aging men.

\section{Dipstick Urinalysis}

The method of conducting dipstick urinalysis differed among the three studies. SSS was conducted in urology offices. Routine in-office dipstick urinalysis was conducted for all men who believed OTC tamsulosin was right for them to use. Men with dipstick urinalysis findings were asked to undergo a full urological assessment to identify potential underlying medical conditions. AUS8 was conducted at retail pharmacy sites. Men who believed OTC tamsulosin was right for them to use underwent dipstick urinalysis prior to product purchase. They were provided with a sterile urine collection kit, which included a container, towelettes, and instructions on how to collect a midstream, clean-catch urine specimen. Dipstick urinalysis was conducted using five-panel Chemstrip $^{\circledR} 5$ OB urine test strips (Roche Diagnostics, Indianapolis, IN, USA), which were machine-read using a Urisys $1100^{\circledR}$ Urine Analyzer (Roche Diagnostics). Urinary blood, glucose, and/or leukocyte results were recorded in the participants' electronic records. For men 
i. Self-selection study (SSS)

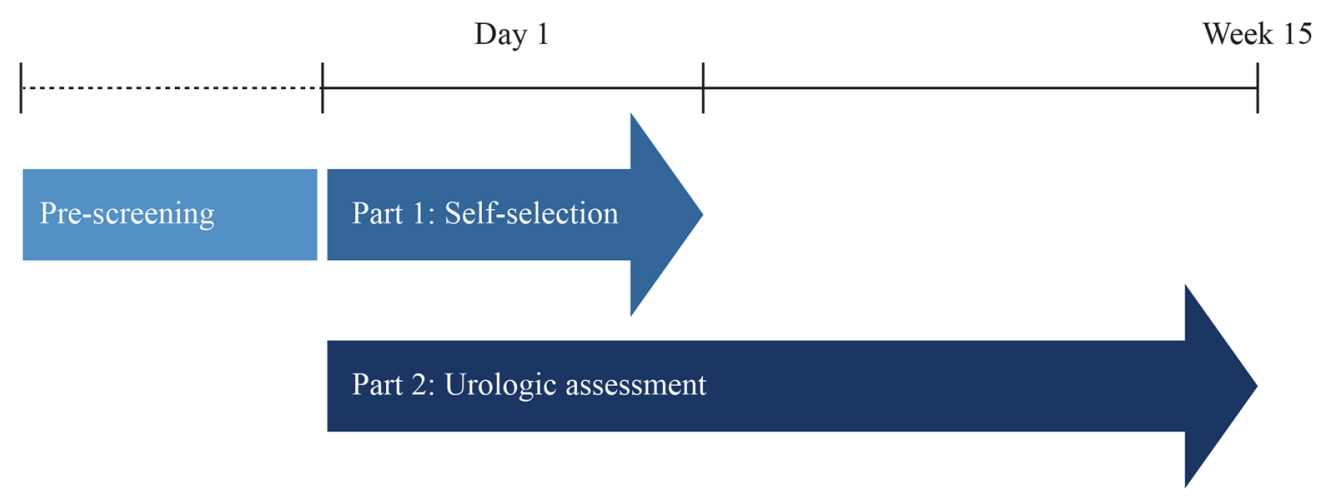

ii. 8-week actual-use study (AUS8)

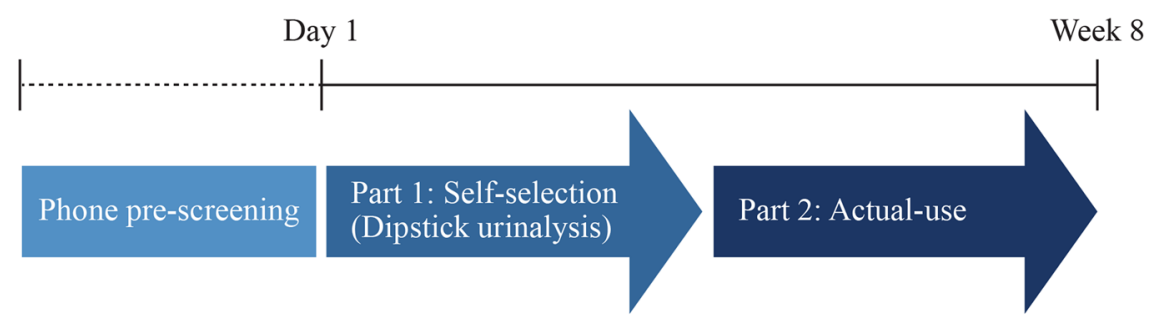

iii. 24-week actual-use study (AUS24)

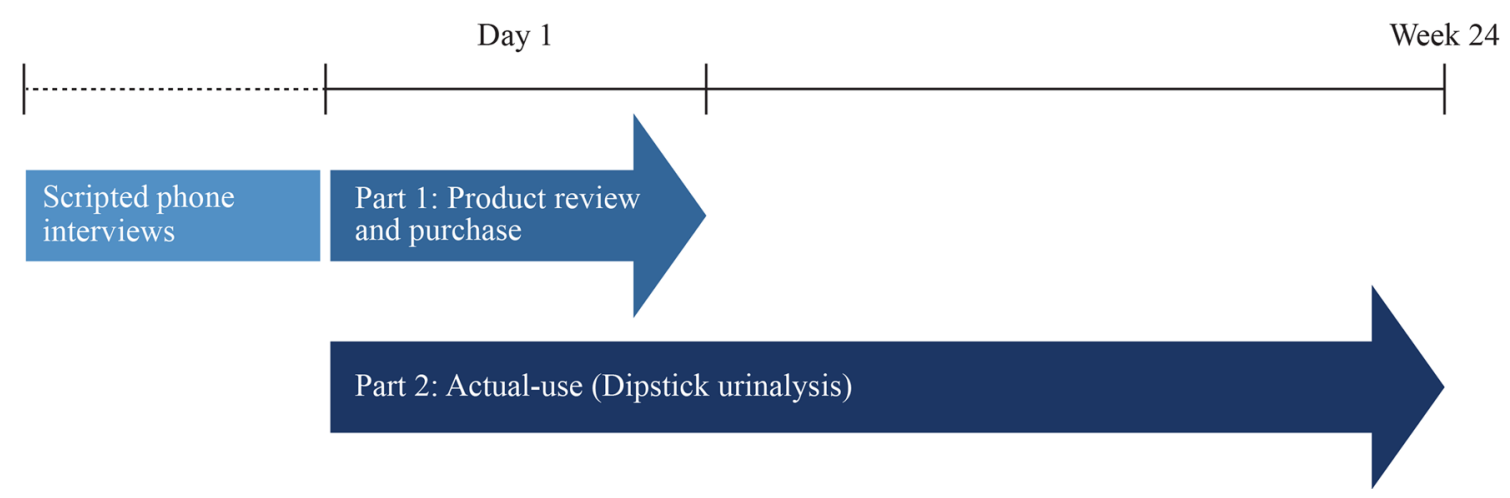

Fig. 1 Flowchart of the SSS, AUS8, and AUS24 studies

with a trace or more of hematuria, glucosuria, and/or leukocyturia, specimens were sent to a local laboratory for culture and sensitivity, and participants were advised to visit a physician. AUS24 was also conducted at retail pharmacy sites. In AUS24, urine sample collection followed a process similar to AUS8, except that pharmacists were asked to also provide verbal instructions on how to properly collect urine. Urine samples were analyzed as described for
AUS8. Dipstick urinalysis was conducted prior to product purchase and was repeated at the end of study for only those men who had a positive dipstick finding at baseline.

\section{Urological Assessment in SSS}

Men with a dipstick finding of a trace or more of hematuria, glucosuria, and/or leukocyturia comprised a predefined subpopulation that was 
asked to undergo urological assessments. Study urologists conducted complete urological assessments using their clinical judgment and any diagnostic testing needed to determine the cause of the dipstick finding as well as the cause of participants' urinary symptoms.

\section{Statistical Analysis}

The overall analysis population comprised nonRx users and Rx users from the three studies. The analysis population comprised men with a dipstick finding of blood, glucose, and/or leukocytes. The safety population comprised men from AUS8 and AUS24 who purchased and took at least one dose of OTC tamsulosin. Descriptive statistics were used to summarize baseline demographics, dipstick urinalysis results, urological assessments, and newly diagnosed MICs. Because of the exploratory nature of the studies, no hypothesis-testing statistical analysis was applied. The analysis was performed using SAS statistical software (SAS v. 9.4, Cary, NC, USA).

\section{RESULTS}

\section{Participant Characteristics}

Overall, 680 nonRx users (mean age \pm standard deviation, $61.4 \pm 11.6$ years) were interested in using OTC tamsulosin and had a positive urine dipstick finding (Table 1$)$. Rx users $(n=678)$ were somewhat older $(64.7 \pm 9.5$ years in SSS $[n=171], 70.7 \pm 9.4$ years in AUS8 [ $n=143]$, and $68.3 \pm 9.1$ years in AUS24 $[n=364])$. The most frequently reported baseline medical conditions by nonRx users and $\mathrm{Rx}$ users, regardless of dipstick findings, were high blood pressure, high cholesterol, diabetes mellitus, and erectile dysfunction (Table 1 and Supplementary Table 1). According to the AUA-Symptom Index total score, a majority of these men were moderately $(45.7 \%)$ or severely $(44.0 \%)$ symptomatic. The proportion of men with dipstick urinalysis findings was similar between men who reported visiting their HCPs at least once a year and those who did not report visiting their HCPs at least once a year (Supplementary Table 2 ).

Overall, 2488 nonRx users who believed that OTC tamsulosin was right for them to use or were interested in purchasing it and $406 \mathrm{Rx}$ users (171 in SSS, 84 in AUS8, and 151 in AUS24) had a baseline urine dipstick test performed. Positive urine dipstick findings were recorded for $680(27.3 \%)$ nonRx users, 37 (21.6\%) Rx users in SSS, 23 (27.4\%) Rx users in AUS8, and 47 (31.1\%) Rx users in AUS24. Table 2 summarizes the specific baseline urine dipstick findings for the three studies.

\section{Outcomes of Urological Assessments in SSS}

We split the data into nonRx and Rx users, as Rx users are not at risk of self-treating urinary symptoms caused by an undiagnosed MIC (the use of $\mathrm{BPH}$ prescription medications assumes that the treating physician has ruled out undiagnosed MICs, whereas this cannot be assumed for all nonRx users). Among 626 interested nonRx users and $103 \mathrm{Rx}$ users who underwent urological assessments by a board-certified urologist, 200 nonRx users and $34 \mathrm{Rx}$ users had a positive urine dipstick finding $(31.9 \%$ and $33.0 \%$, respectively; Table 3). Overall, urologists identified $130(20.8 \%)$ nonRx users and 10 (9.7\%) Rx users with newly diagnosed urological or nonurological conditions that could cause or contribute to urinary symptoms; among these, 26 nonRx users and $1 \mathrm{Rx}$ user had a positive urine dipstick finding. Most of these conditions were not labeled as MICs. Table 3 summarizes the number of nonRx users and Rx users with newly diagnosed conditions. The MICs identified in nonRx users with a positive dipstick finding included prostate cancer $(1.0 \%)$, urolithiasis (1.0\%), bladder tumor $(0.5 \%)$, diabetes mellitus $(0.5 \%)$, urinary incontinence $(0.5 \%)$, and urinary retention $(0.5 \%)$.

\section{MICs Reported in AUS}

Overall, 1265 nonRx users in the safety population had undergone urine dipstick testing and $401(31.7 \%)$ had a positive dipstick finding 


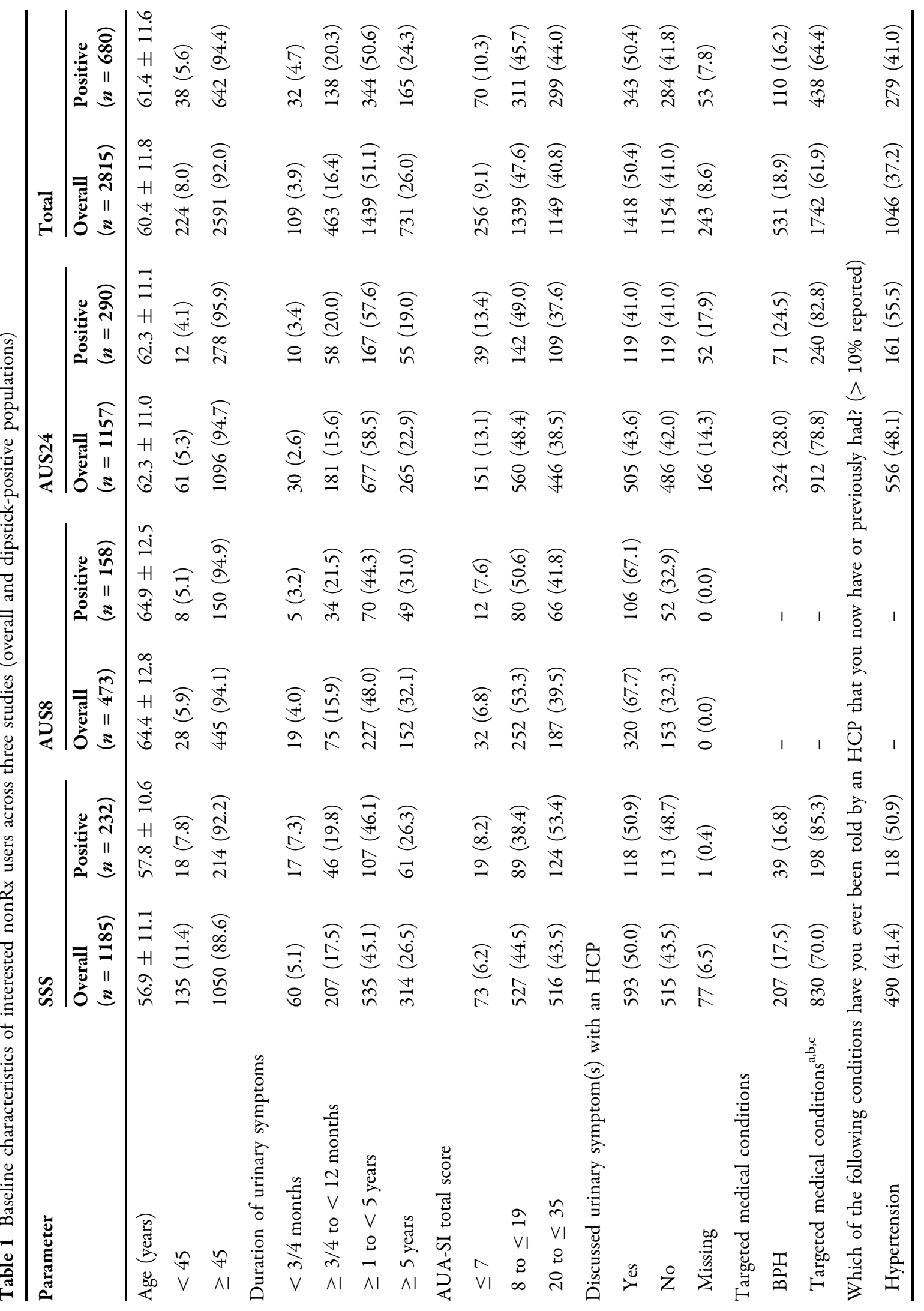




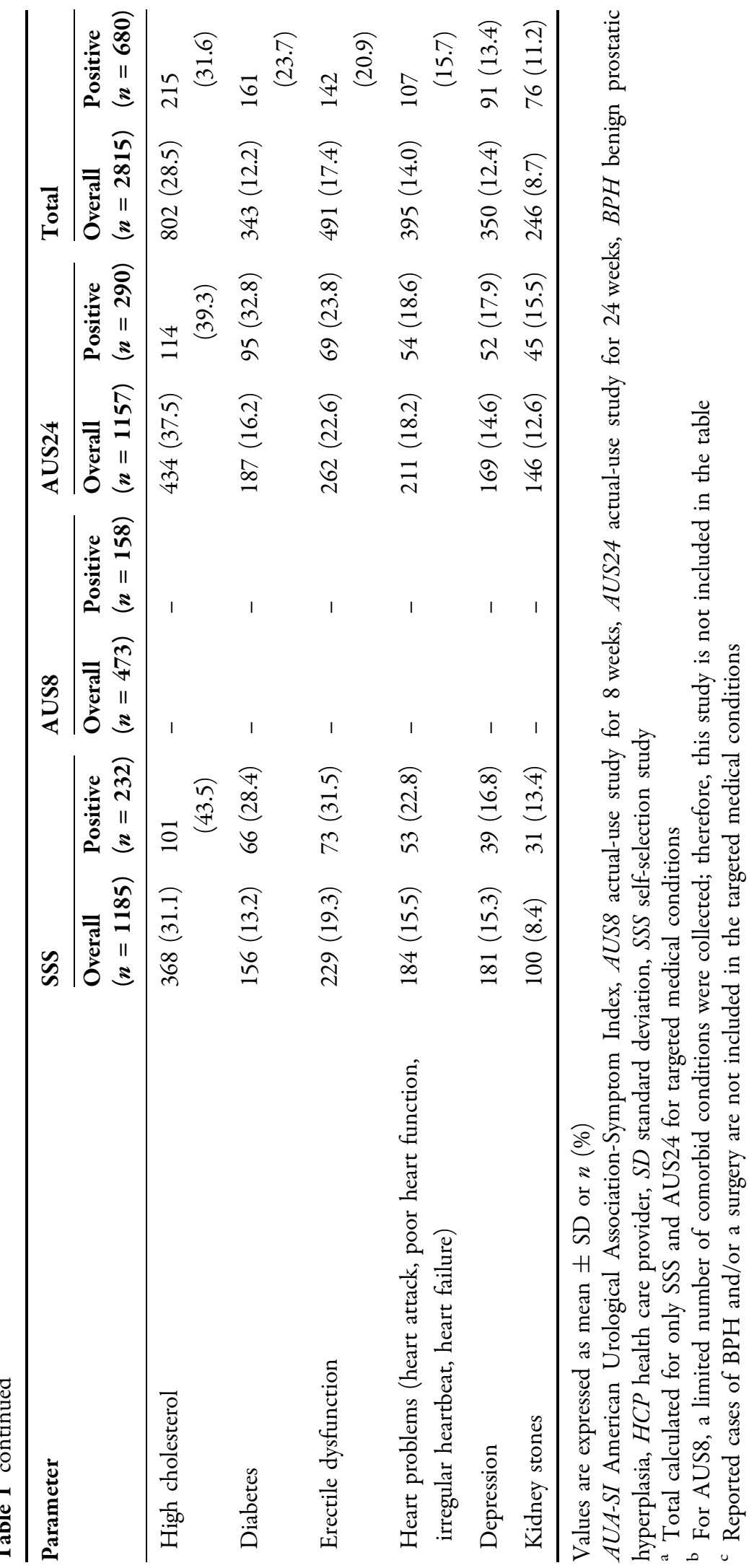


Table 2 Baseline dipstick urinalysis findings in nonRx users and Rx users

\begin{tabular}{|c|c|c|c|c|c|c|c|}
\hline & \multicolumn{4}{|l|}{ NonRx users } & \multicolumn{3}{|l|}{ Rx users } \\
\hline & $\begin{array}{l}\text { SSS } \\
(n=1185)\end{array}$ & $\begin{array}{l}\text { AUS8 } \\
(n=473)\end{array}$ & $\begin{array}{l}\text { AUS24 } \\
(n=1157)\end{array}$ & $\begin{array}{l}\text { Total } \\
(n=2815)\end{array}$ & $\begin{array}{l}\text { SSS } \\
(n=171)\end{array}$ & $\begin{array}{l}\text { AUS8 } \\
(n=84)\end{array}$ & $\begin{array}{l}\text { AUS24 } \\
(n=225)\end{array}$ \\
\hline $\begin{array}{l}\text { Men who underwent } \\
\text { testing }\end{array}$ & $1116(100.0)$ & $403(100.0)$ & $969(100.0)$ & $2488(100.0)$ & $171(100.0)$ & $84(100.0)$ & $151(100.0)$ \\
\hline $\begin{array}{l}\text { Men with positive } \\
\text { findings }\end{array}$ & $232(20.8)$ & $158(39.2)$ & $290(29.9)$ & $680(27.3)$ & $37(21.6)$ & $23(27.4)$ & $47(31.1)$ \\
\hline Glucosuria & $72(6.5)$ & $79(19.6)$ & $108(11.1)$ & $259(10.4)$ & $9(5.3)$ & $7(8.3)$ & $21(13.9)$ \\
\hline Hematuria & $143(12.8)$ & $66(16.4)$ & $123(12.7)$ & $332(13.3)$ & $20(11.7)$ & $10(11.9)$ & $15(9.9)$ \\
\hline Leukocyturia & $55(4.9)$ & $73(18.1)$ & $117(12.1)$ & $245(9.8)$ & $12(7.0)$ & $15(17.9)$ & $19(12.6)$ \\
\hline
\end{tabular}

Values are expressed as $n$ (\% of men who underwent testing). A given patient could be positive for more than one finding. Data from Rx users were not integrated and thus cannot be displayed as totals

AUS8 actual-use study for 8 weeks, AUS24 actual-use study for 24 weeks, SSS self-selection study

(Table 4). In the nonRx group, the percentage of participants with newly diagnosed MICs was similar between participants with and without positive urine dipstick findings except for diabetes and urinary tract infection. The newly diagnosed conditions or exacerbations of an MIC reported among the nonRx users with a positive urine dipstick finding were urinary tract infection (3.0\%), diabetes mellitus (2.5\%), urinary incontinence (1.2\%), and hypertension (1.0\%; Table 4).

\section{DISCUSSION}

$\mathrm{BPH} / \mathrm{BPE}$ is the most common cause of male LUTS, but this condition is multifactorial. Guidelines recommend the use of dipstick urinalysis to screen for undiagnosed non-BPH/BPE MICs that can cause or contribute to male LUTS $[1,2]$. We have analyzed data from three recently published studies [9-11] to broaden the evidence base on the utility of dipstick urinalysis to screen men presenting with LUTS for pathologies other than or in addition to $\mathrm{BPH} /$ BPE. The three analyzed studies were based on men considering the use of an OTC tamsulosin product, which may have led to selection bias.

Positive findings were similarly common among the pooled nonRx users, likely to include many men currently not undergoing professional care for their LUTS, and in Rx users, by definition currently receiving LUTS medication from a physician (27.3\% vs. $21.6-31.1 \%)$. Differences in the prevalence of positive dipstick findings were probably related to those in comorbidities in the three study populations, i.e., positive dipstick findings were more frequent in the studies with greater prevalence of known comorbidities. Accordingly, the rationale for routine dipstick screening of men presenting with LUTS would be similar to that for screening of all men visiting a physician for any reason, a suggestion not supported by any major guideline.

As reported previously for SSS $(2.9 \%$ MICs) [10], relatively few newly diagnosed MICs were identified during follow-up urological assessments. Moreover, MICs were identified with similar incidence in participants with and without positive dipstick findings in the two actual-use studies, the only possible exceptions being urinary tract infection and diabetes (Table 4). The diagnosis of urinary tract infection or diabetes requiring immediate medical attention is based on clinical symptoms in routine care, not on a general dipstick screening, again supporting the hypothesis that the rationale for routine dipstick screening of men presenting with LUTS is not stronger than that for screening of the general population. Therefore, we focused on the detection of previously 
Table 3 Number of men with newly diagnosed urological and nonurological conditions causing or contributing to urinary symptoms in SSS

\begin{tabular}{|c|c|c|c|c|}
\hline & $\begin{array}{l}\text { NonRx } \\
\text { users }\end{array}$ & Rx users & $\begin{array}{l}\text { NonRx users with positive } \\
\text { urine dipstick findings }\end{array}$ & $\begin{array}{l}\mathrm{Rx} \text { users with positive } \\
\text { urine dipstick findings }\end{array}$ \\
\hline Number of men & 1103 & 171 & 229 & 37 \\
\hline Men who underwent testing & $\begin{array}{l}626 \\
(100.0)\end{array}$ & $\begin{array}{l}103 \\
(100.0)\end{array}$ & $200(100.0)$ & $34(100.0)$ \\
\hline $\begin{array}{l}\text { Men with other urological or } \\
\text { nonurological conditions }\end{array}$ & $\begin{array}{l}130 \\
(20.8)\end{array}$ & $10(9.7)$ & $26(13.0)$ & $1(2.9)$ \\
\hline \multicolumn{5}{|l|}{ Medically important conditions } \\
\hline $\begin{array}{l}\text { Bladder tumor, not otherwise } \\
\text { specified }\end{array}$ & $3(0.5)$ & - & $1(0.5)$ & - \\
\hline Diabetes mellitus & $2(0.3)$ & - & $1(0.5)$ & - \\
\hline Hypertension & $1(0.2)$ & - & - & - \\
\hline Prostate cancer & $6(1.0)$ & - & $2(1.0)$ & - \\
\hline $\begin{array}{l}\text { Prostate tumor of uncertain } \\
\text { behavior }\end{array}$ & $2(0.3)$ & - & - & - \\
\hline Urinary incontinence & $1(0.2)$ & - & $1(0.5)$ & - \\
\hline Urinary retention & $1(0.2)$ & - & $1(0.5)$ & - \\
\hline Urinary tract infection & $1(0.2)$ & - & - & - \\
\hline Urolithiasis & $2(0.3)$ & $1(1.0)$ & $2(1.0)$ & $1(2.9)$ \\
\hline \multicolumn{5}{|l|}{ Other conditions } \\
\hline Anxiety & $1(0.2)$ & - & - & - \\
\hline Benign microscopic hematuria & $2(0.3)$ & - & $2(1.0)$ & - \\
\hline Bladder outlet obstruction & $16(2.6)$ & - & $2(1.0)$ & - \\
\hline $\mathrm{BPH}$ & $1(0.2)$ & - & - & - \\
\hline Dietary irritants & $12(1.9)$ & $1(1.0)$ & $2(1.0)$ & - \\
\hline $\begin{array}{l}\text { Medication-induced urinary } \\
\text { symptoms }\end{array}$ & $1(0.2)$ & - & $1(0.5)$ & - \\
\hline Neurogenic voiding dysfunction & $13(2.1)$ & $1(1.0)$ & $3(1.5)$ & - \\
\hline Nocturia & $1(0.2)$ & $1(1.0)$ & - & - \\
\hline Non-BPH LUTS & $1(0.2)$ & - & - & - \\
\hline Overactive bladder & $37(5.9)$ & $2(1.9)$ & $4(2.0)$ & - \\
\hline Peripheral edema & $1(0.2)$ & - & - & - \\
\hline Polydipsia & $16(2.6)$ & $4(3.9)$ & $2(1.0)$ & - \\
\hline Prostatitis & $19(3.0)$ & - & $2(1.0)$ & - \\
\hline
\end{tabular}


Table 3 continued

\begin{tabular}{lllll}
\hline & $\begin{array}{l}\text { NonRx } \\
\text { users }\end{array}$ & $\mathbf{R x}$ users & $\begin{array}{l}\text { NonRx users with positive } \\
\text { urine dipstick findings }\end{array}$ & $\begin{array}{l}\text { Rx users with positive } \\
\text { urine dipstick findings }\end{array}$ \\
\hline $\begin{array}{l}\text { Renal cystic disease } \\
\begin{array}{l}\text { Renal tumor, not otherwise } \\
\text { specified }\end{array}\end{array}$ & $1(0.2)$ & - & $1(0.5)$ & - \\
$\begin{array}{l}\text { Sleep apnea } \\
\text { Urethritis }\end{array}$ & $2(0.3)$ & - & $1(0.5)$ & - \\
$\begin{array}{l}\text { Voiding dysfunction } \\
\begin{array}{l}\text { Post-prostatectomy voiding } \\
\text { dysfunction, not otherwise }\end{array}\end{array}$ & $1(0.2)$ & - & - & - \\
specified
\end{tabular}

Patients may be included in more than one category. Dash (-) indicates that none of the patients had the condition $B P H$ benign prostatic hyperplasia, LUTS lower urinary tract symptoms, SSS self-selection study

undetected prostate or bladder cancer following a positive urine dipstick finding.

Notably, only $0.2 \%$ of men with positive urine dipstick findings had a new diagnosis or exacerbation of prostate or bladder cancer in our analysis. In a South Korean study of 56,632 participants in a general health-screening program (i.e., not selecting for men with LUTS), $6.2 \%$ of participants had AMH at initial urinalysis. Of these, $56.3 \%$ tested positive at the 1-year repeat urinalysis and only $3.7 \%$ had abnormal lesions as underlying diseases, most commonly urinary stones [12]. In another study, 258 men at least 50 years old were identified from well-patient clinic rosters as having $\mathrm{AMH}$ detected by home testing of their urine by using chemical reagent strips for hemoglobin. These men subsequently tested their urine repeatedly according to one of the two protocols: five daily testings followed by one testing per week for a year, or 14 daily testings at the beginning of the study and 9 months later. Of 234 men with initial negative evaluations for urinary tract cancers, only $2(0.85 \%)$ developed bladder cancer during the 14-year follow-up period [13]. In a single-center study from Scotland, 16 of 292 patients with a positive dipstick finding of AMH had urological malignancies, 33 had a non-malignant cause of $\mathrm{AMH}$, and 180 failed to confirm AMH upon repeat testing [14].
Thus, very few cases of prostate or bladder cancer are newly detected in the screening of men with LUTS or in the general population.

It could be argued that use of routine dipstick urinalysis is warranted even if only very few cases of prostate or bladder cancer are newly detected because the test is relatively cheap. However, the costs and inconveniences of the additional investigations triggered by a positive dipstick finding are more substantial. In previous studies, use of dipstick urinalysis screening in men with LUTS and subsequent false-positive results were implicated in unnecessary and costly urological workup [15-17]. Moreover, patients may be exposed to risks such as radiation during imaging or development of infections during cystoscopy [15]. Collectively, these findings suggest that current practices for evaluating men with LUTS can contribute to financial burden on the health care system and expose patients to needless procedures [16].

Two studies have addressed the cost-effectiveness of common diagnostic approaches recommended by the AUA guidelines for AMH management [18, 19]. Using a decision tree model, Bandari et al. compared the cost-effectiveness of an upfront approach (immediate $\mathrm{AMH}$ evaluation) based on a single positive urinalysis with a confirmatory approach (delayed evaluation requiring a confirmatory 
Table 4 Medically important conditions in nonRx users in AUS8 and AUS24 (safety population)

\begin{tabular}{llc}
\hline & $\begin{array}{l}\text { Men without positive urine } \\
\text { dipstick findings } \\
\boldsymbol{n}=\mathbf{8 6 4}(\mathbf{1 0 0 . 0})\end{array}$ & $\begin{array}{l}\text { Men with positive urine } \\
\text { dipstick findings } \\
\boldsymbol{n}=\mathbf{4 0 1}(\mathbf{1 0 0 . 0})\end{array}$ \\
\hline Bladder cancer & $0(0.0)$ & $1(0.2)$ \\
Diabetes mellitus & $0(0.0)$ & $10(2.5)$ \\
Hypertension & $4(0.5)$ & $4(1.0)$ \\
Prostate cancer & $1(0.1)$ & $1(0.2)$ \\
Type 2 diabetes mellitus & $0(0.0)$ & $1(0.2)$ \\
Urinary incontinence & $6(0.7)$ & $5(1.2)$ \\
Urinary retention & $1(0.1)$ & $0(0.0)$ \\
Urinary tract infection & $1(0.1)$ & $12(3.0)$ \\
\hline
\end{tabular}

Shown here are all conditions being diagnosed as part of the workup of the participants, irrespective of a plausible pathophysiological relationship with the dipstick findings. Values are expressed as $n(\%)$. Percentages are calculated using total number of participants per treatment as the denominator. Medically significant conditions are listed by system organ class/preferred term (MedDRA version 19.1). For AUS8, the treatment period was defined as the self-selection date to end of study date +7 days. For AUS24, the treatment period was defined as the purchase of study drug date to end of study date +3 days

AUS8 actual-use study for 8 weeks, AUS24 actual-use study for 24 weeks

positive urinalysis result) [18]. Results showed that the upfront approach was $66 \%$ more costly than the confirmatory approach $(\$ 776$ vs. $\$ 466 /$ patient), with an incremental cost-effectiveness ratio of $\$ 10,719 /$ life-year gained; the willingness to pay threshold was set at $\$ 100,000 /$ life-year. However, the authors concluded that the confirmatory approach is favorable over the upfront approach only if there is a low possibility of delayed cancer detection or minimal loss of life expectancy associated with a delayed diagnosis [18]. In another study using a decision-analytic model, four diagnostic approaches were compared with no evaluation [19]. The combination of renal ultrasonography and cystoscopy was deemed the most cost-effective approach for initial evaluation of $\mathrm{AMH}$ when cancer detection was considered the primary outcome. Furthermore, guidelines do not risk-stratify patients for evaluation. Overall, these results suggest that the current guidelines need to be critically evaluated to determine the most clinically effective and cost-effective screening strategies.
In view of the existing variations in the management and workup of patients with hematuria, a group of Kaiser Permanente urology chiefs proposed national practice recommendations for hematuria to avoid unnecessary evaluations in low-risk patients [20]. According to these recommendations, only patients with gross hematuria or high-grade hematuria $(>50$ red blood cells [RBCs]/high-power field [HPF] on a single microscopic urinalysis) or patients with $\mathrm{AMH}$ or symptomatic hematuria and more than 3 RBCs/HPF on two of three accurately performed and collected urinalyses should be referred to urologists for urological evaluations. Furthermore, routine urinalysis screening of adults for AMH is not recommended because of the low incidence and intermittent occurrence of significant associated urological disease [20]. The AUA panel defines microhematuria as an average of at least $3 \mathrm{RBCs} / \mathrm{HPF}$ in two of three properly collected urine specimens [8]. Authors of a review on the evaluation of hematuria suggested that only patients with documented true hematuria based on the AUA guidelines 
should be referred for further evaluation to avoid expensive unnecessary testing [16].

While results of this analysis help to clarify the benefit (or lack thereof) of dipstick urinalysis, some limitations must be acknowledged. First, positive dipstick results were verified by further testing only in SSS, and the rates of falsepositive results in AUS8 and AUS24 were not assessed. Further, patients in the analysis were not stratified by potential risk factors for MICs, such as age, smoking status, or degree of hematuria. Regardless of the limitations, the current evidence on the use of dipstick urinalysis in men with LUTS does not seem to support its utility as a routine screening tool for undiagnosed MICs. The most important limitation of our analysis is that it is a post hoc analysis based on three studies not originally intended to determine the utility of routine dipstick use. Therefore, results of this analysis cannot be seen as definitive proof that routine dipstick analysis should be abandoned. However, the results raise sufficient doubt on the guideline-recommended practice $[5,8]$ and therefore we strongly recommend prospective dedicated studies for the routine use of dipstick urinalysis in patients with LUTS. This may lead to more cost-effective medical care and avoid morbidity caused by follow-up investigation in case of positive dipstick findings.

\section{CONCLUSION}

Results of this analysis show that use of dipstick urinalysis identifies relatively few undiagnosed, non-BPH/BPE MICs that could account for urinary symptoms. Therefore, dipstick urinalysis may not be an effective screening tool in men with bothersome LUTS.

\section{ACKNOWLEDGEMENTS}

We thank all participants of the underlying studies.

Funding. The underlying studies and this analysis were funded by Boehringer Ingelheim
Pharmaceuticals, Inc. (BIPI). No Rapid Service Fee was received by the journal for the publication of this article. BIPI funded the open access fee for this article. All authors had full access to all of the data in this study and take complete responsibility for the integrity of the data and accuracy of the data analysis.

Medical Writing and Editorial Assistance. Writing, editorial support and formatting assistance was provided by Suchita NathSain, Ph.D., and Maribeth Bogush, Ph.D., of Cactus Communications, who were contracted and compensated by BIPI for these services. Boehringer Ingelheim Pharmaceuticals, Inc. was given the opportunity to review the manuscript for medical and scientific accuracy as well as intellectual property considerations.

Authorship. All named authors meet the International Committee of Medical Journal Editors (ICMJE) criteria for authorship for this article, take responsibility for the integrity of the work as a whole, and have given their approval for this version to be published.

Disclosures. Franklin Lowe was a consultant for Boehringer Ingelheim at the time the underlying studies were performed and is now affiliated with Weiler Hospital, Albert Einstein College of Medicine, Bronx, NY, USA. Martin C. Michel was an employee of Boehringer Ingelheim at the time the study was conducted and, outside of the submitted work, received personal fees from Apogepha, Astellas, Ferring, Recordati, Dr. Willmar Schwabe and grants from Velicept. Martin C. Michel is now affiliated with Johannes Gutenberg University, Obere Zahlbacher, Mainz, Germany and is a member of the journal's Editorial Board. Jan Wruck is an employee of Boehringer Ingelheim. Anna Verbeek was an employee of Boehringer Ingelheim at the time the study was conducted and is currently an employee of Sanofi.

Compliance with Ethics Guidelines. The published underlying studies were in accordance with the ethical standards of the institutional and/or national research committee and with the 1964 Helsinki Declaration and its later 
amendments or comparable ethical standards. Informed consent was obtained from all individual participants included in the studies. Ethics committee approval was not applicable to the post hoc analysis reported in the present manuscript. The underlying studies were approved by independent ethics committees which are detailed in the previous publications.

Data Availability. The datasets generated during and/or analyzed during the current study are available from the corresponding author on reasonable request.

Open Access. This article is distributed under the terms of the Creative Commons Attribution-NonCommercial 4.0 International License (http://creativecommons.org/licenses/ by-nc/4.0/), which permits any noncommercial use, distribution, and reproduction in any medium, provided you give appropriate credit to the original author(s) and the source, provide a link to the Creative Commons license, and indicate if changes were made.

\section{REFERENCES}

1. American Urological Association guideline: Management of benign prostatic hyperplasia (BPH), 2010. http://www.auanet.org/guidelines/benignprostatic-hyperplasia-(2010-reviewed-and-validityconfirmed-2014). Accessed April 24, 2019.

2. Gravas S, Cornu JN, Drake MJ, et al. EAU guidelines on the management of non-neurogenic male lower urinary tract symptoms including benign prostatic obstruction. 2018. https://uroweb.org/wp-content/ uploads/EAU-Guidelines-on-the-Management-ofNon-neurogenic-Male-LUTS-2018-large-text.pdf. Accessed April 24, 2019.

3. Fourcade RO, Lacoin F, Roupret M, et al. Outcomes and general health-related quality of life among patients medically treated in general daily practice for lower urinary tract symptoms due to benign prostatic hyperplasia. World J Urol. 2012;30:419-26.

4. Khasriya R, Khan S, Lunawat R, et al. The inadequacy of urinary dipstick and microscopy as surrogate markers of urinary tract infection in urological outpatients with lower urinary tract symptoms without acute frequency and dysuria. J Urol. 2010;183:1843-7.

5. Simerville JA, Maxted WC, Pahira JJ. Urinalysis: a comprehensive review. Am Fam Physician. 2005;71:1153-62.

6. Pallin DJ, Ronan C, Montazeri K, et al. Urinalysis in acute care of adults: pitfalls in testing and interpreting results. Open Forum Infect Dis. 2014;1(1):ofu019.

7. Deville WL, Yzermans JC, van Duijn NP, et al. The urine dipstick test useful to rule out infections. A meta-analysis of the accuracy. BMC Urol. 2004;4:4.

8. Davis R, Jones JS, Barocas DA, et al. Diagnosis, evaluation and follow-up of asymptomatic microhematuria $(\mathrm{AMH})$ in adults: AUA guideline. J Urol. 2012; 188:2473-81.

9. Roehrborn CG, Lowe FC, Gittelman M, et al. Management of male lower urinary tract symptoms in a simulated over-the-counter setting: an exploratory, study of tamsulosin. Drugs Aging. 2019;36:179-88.

10. Roehrborn CG, Lowe FC, Gittelman M, et al. Feasibility of an alternative option for the management of male lower urinary tract symptoms. J Urol. 2016;195:125-30.

11. Roehrborn CG, Lowe FC, Gittelman M, et al. Simulated over-the-counter use of tamsulosin by men with urinary symptoms. J Sex Med. 2018;15:S1.

12. Kang M, Lee S, Jeong SJ, et al. Characteristics and significant predictors of detecting underlying diseases in adults with asymptomatic microscopic hematuria: a large case series of a Korean population. Int J Urol. 2015;22:389-93.

13. Madeb R, Golijanin D, Knopf J, et al. Long-term outcome of patients with a negative work-up for asymptomatic microhematuria. Urology. 2010;75:20-5.

14. Mishriki SF, Nabi G, Cohen NP. Diagnosis of urologic malignancies in patients with asymptomatic dipstick hematuria: prospective study with 13 years' follow-up. Urology. 2008;71:13-6.

15. Rao PK, Jones JS. How to evaluate 'dipstick hematuria': what to do before you refer. Cleve Clin J Med. 2008;75:227-33.

16. Rao PK, Gao T, Pohl M, et al. Dipstick pseudohematuria: unnecessary consultation and evaluation. J Urol. 2010;183:560-4.

17. Samal L, Linder JA. The primary care perspective on routine urine dipstick screening to identify patients 
with albuminuria. Clin J Am Soc Nephrol. 2013;8:131-5.

18. Bandari J, Jacobs B, Smith K. Evaluating the cost effectiveness of the asymptomatic microhematuria guidelines. J Urol. 2017;197:e281.

19. Halpern J, Chughtai B. Cost-effectiveness of common diagnostic approaches for evaluation of asymptomatic microscopic hematuria. J Urol. 2017;197:e280.

20. Loo R, Whittaker J, Rabrenivich V. National practice recommendations for hematuria: how to evaluate in the absence of strong evidence? Perm J. 2009;13:37-46. 